\title{
Synchronization in a coupled two-layer quasigeostrophic model of baroclinic instability - Part 1: Master-slave configuration
}

\author{
A. A. Castrejón-Pita and P. L. Read \\ Atmospheric, Oceanic \& Planetary Physics, Clarendon Laboratory, University of Oxford, Parks Road, Oxford, OX1 3PU, UK
}

Received: 27 March 2009 - Revised: 15 July 2009 - Accepted: 4 August 2009 - Published: 7 August 2009

\begin{abstract}
Synchronization is studied using a pair of diffusively-coupled, two-layer quasi-geostrophic systems each comprising a single baroclinic wave and a zonal flow. In particular, the coupling between the systems is in the well-known master-slave or one-way configuration. Nonlinear time series analysis, phase dynamics, and bifurcation diagrams are used to study the dynamics of the coupled system. Phase synchronization, imperfect synchronization (phase slips), or complete synchronization are found, depending upon the strength of coupling, when the systems are either in a periodic or a chaotic regime. The results of investigations when the dynamics of each system are in different regimes are also presented. These results also show evidence of phase synchronization and signs of chaos control.
\end{abstract}

\section{Introduction}

For several years, regular fluctuations in climate dynamics have intrigued climate scientists and researchers. Some investigations have been performed to demonstrate the cyclic behavior in the Earth climate such as the well-known El Niño phenomenon, the index cycle, and the North Atlantic Oscillation (Tziperman et al., 1995). It is often unclear, however, whether this cyclic behaviour is produce by external periodic forcing (solar forcing for instance), internal variability, or some interaction between the two (Gildor and Tziperman, 2000).

Certain fluctuations in meteorological records are also characterized by teleconnection patterns in both space and time, in the sense of significant correlations between the fluctuations of a field at widely separated points, commonly applied to variability on monthly and longer timescales. Such

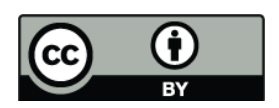

Correspondence to: A. A. Castrejón-Pita (aacp@atm.ox.ac.uk) correlations suggest that some kind of information may be propagated between two (or more) distant places through the atmosphere. For instance, teleconnections between middle latitude blocking of the Northern and Southern Hemispheres have been diagnosed in atmospheric data (e.g. Duane et al., 1999). These teleconnections are manifested as a small but significant tendency for blocking to occur simultaneously in the two hemispheres. Ideas of chaos synchronization have been used to explain this phenomenon (Duane et al., 1999; Duane, 1997). In those investigations, it was found that, even though the events considered singly in each hemisphere are chaotic in time, correlations between blocking events in the Northern and Southern Hemispheres were taking place, (Duane and Tribbia, 2001). Similar results have also been found and studied using global GCMs or coupled-hemisphere models (Lunkeit, 2001). Phase synchronization between atmospheric variables, such as daily mean temperature and daily precipitation records, has also been studied by Rybski et al. (2003).

The study of synchronization in simple experiments and simplified models can provide a useful source of insight for these atmospheric phenomena and more generally in the study of climate variability. Since the first studies of baroclinic waves in the atmosphere, the two-layer model has played an important role. It was proposed more than 50 years ago to encapsulate in the simplest way some of the principal features of the middle latitude atmospheric circulation. Since then, it has been widely used in studies of baroclinic instability (Phillips, 1954; Pedlosky, 1970, 1971, 1972), and it has also been used as an idealization of the rotating annulus laboratory experiment (Hide and Mason, 1975; Lovegrove et al., 2001).

In this paper, we use a five-dimensional version of the two-layer, minimal quasigeostrophic model of baroclinic instability presented in Lovegrove et al. $(2001,2002)$ and Eccles et al. (2006). This model can be understood as one of the simplest models to represent non-linear dynamics of the

Published by Copernicus Publications on behalf of the European Geosciences Union and the American Geophysical Union. 
baroclinic instability of a single wave in the presence of a baroclinic zonal flow. Although it is clear that this model is far removed from realistic atmospheric situations, it can reproduce several of the basic dynamical regimes found in rotating annulus experiments. Those include experiments in real two layer systems, (Appleby, 1982; Lovegrove, 1997) or the thermally driven rotating baroclinic annulus (Hide and Mason, 1975; Read et al., 1992, 1998).

As shown in Lovegrove et al. (2001) and Lovegrove et al. (2002), when subject to steady boundary conditions this simple model exhibits a rich structure of bifurcations, including period doubling cascades, leading to regimes corresponding to stationary waves, traveling waves, amplitude vacillating waves (AV), and chaotic modulated amplitude vacillating waves (MAV). This model has also been previously used to study the effects of external periodic forcing on the dynamics by Eccles et al. (2006), who found frequency locking between the natural oscillation frequencies and the forcing frequencies under certain conditions.

The main objective of this paper is to present results of investigations carried out using a coupled pair of such twolayer models. The coupling between the two systems under study in this paper is in the one-way or unidirectional configuration (better known as master-slave configuration). This connection is achieved by adding a linear diffusive coupling term into the mean flow correction equation of the system, being the most natural and simplest way to introduce a zonally symmetric interaction between the two systems. The organization of the article is as follows. Section 2 provides a general description and formulation of the simple two-layer quasigeostrophic model. Then, in Sect. 3, the modifications performed in order to create a coupled two-layer quasigeostrophic model will be discussed followed by the methods used to analyze the data in Sect. 4. Section 5 presents both general and particular results for each of the main flow types found in the model, showing the kind of synchronized states that can be reached with this system. Finally, Sect. 6 presents a discussion of the results and final remarks.

\section{The model}

The model used here is an adaptation of the 5-dimensional (5-D) model described by Lovegrove et al. (2001), Lovegrove et al. (2002), and Eccles et al. (2006), and the reader is referred to these papers for a more detailed description. The basic model is a two-layer quasi-geostrophic system, formulated in cartesian geometry and permits just one zonally propagating wave with barotropic and baroclinic components. Hence it is arguably the simplest possible representation of such a system.

Following e.g. Salmon (1998), the quasi-geostrophic equations for a two-layer fluid of equal layer depths may be written in terms of barotropic and baroclinic streamfunctions, $\psi_{s}$ and $\psi_{d}$ respectively, (subscript $s$ will henceforth be used for barotropic variables and $d$ for baroclinic) where in our case

$$
\begin{aligned}
& \psi_{s}=\left(\psi_{1}+\psi_{2}\right) / 2, \\
& \psi_{d}=\left(\psi_{1}-\psi_{2}\right) / 2 .
\end{aligned}
$$

In the same way, the barotropic and baroclinic velocities, $U_{d}$ and $U_{s}$, are defined as

$U_{s}=\left(u_{1}+u_{2}\right) / 2$,
$U_{d}=\left(u_{1}-u_{2}\right) / 2$.

The streamfunctions are then projected onto a series of Fourier modes see (Lovegrove et al., 2001, 2002; Lovegrove, 1997):

$$
\begin{aligned}
& \psi_{s, d}=\underbrace{\sum_{m=1}^{M} X_{m}^{s, d} \cos l_{m} y}_{\text {mean flow correction }}+ \\
& \underbrace{\sum_{\substack{n=-N \\
n \neq 0}}^{N} \sum_{m=1}^{M} W_{n m}^{s, d} \exp \left[i k_{n} x\right] \sin l_{m} y}_{\text {wave term }}-\underbrace{U_{s, d} y}_{\text {background }},
\end{aligned}
$$

where

$$
W_{-m n}^{s, d}=\left(W_{m n}^{s, d}\right)^{*} \quad \text { and } \quad k_{-n}=-k_{n} .
$$

The asterisk signifies complex conjugation. The first term on the right hand side of Eq. (3), without any dependence on $x$, is known as the mean flow correction term. This term will be the main focus of our study. The second term is called the wave term and the remainder is the background flow.

\subsection{The 5-dimensional system}

Substituting Eq. (3) into the corresponding quasigeostrophic potential vorticity equations (Lovegrove et al., 2001), and truncating to a single zonal wavenumber term in each of the radial and zonal directions, i.e. $M=N=1$ we obtain

$$
\begin{aligned}
& \dot{A}_{s}=-\Delta_{s} A_{s}+\beta_{s} B_{s}-\left(v_{s}+\gamma_{s} X_{d}\right) B_{d}, \\
& \dot{B}_{s}=-\Delta_{s} B_{s}-\beta_{s} A_{s}+\left(v_{s}+\gamma_{s} X_{d}\right) A_{d}, \\
& \dot{A}_{d}=-\Delta_{d} A_{d}+\beta_{d} B_{d}-\left(v_{d}+\gamma_{d} X_{d}\right) B_{s}, \\
& \dot{B}_{d}=-\Delta_{d} B_{d}-\beta_{d} A_{d}+\left(v_{d}+\gamma_{d} X_{d}\right) A_{s}, \\
& \dot{X}_{d}=-\bar{\Delta} X_{d}+\bar{\gamma}\left[A_{s} B_{d}-B_{s} A_{d}\right],
\end{aligned}
$$

where the over-dot represents differentiation with respect to time, $A_{s, d}=\operatorname{Re}\left(W_{11}^{s, d}\right)$, and $B_{s, d}=-\operatorname{Im}\left(W_{11}^{s, d}\right)$. This system describes the interaction between one azimuthal wave and the mean flow and, whilst not being strictly justifiable physically, it serves to form the simplest possible conceptual model for initial analysis which captures some of the essential qualitative behavior of the full system. The dynamics that this simple model is able to reproduce (when compared to annulus experiments) are, 
- The steady wave regime (Labeled S in Chapter II of this thesis),

- The amplitude vacillation (periodic or quasi-periodic modulated wave) regime (Labeled AV),

- The modulated amplitude vacillation regime (labeled MAV, which can be periodic or chaotic).

Taking into account those results, the dynamics and the effects of coupling two of such systems in those different regimes will be investigated.

\section{The coupled two-layer model}

\subsection{One way coupling}

In recent years, different studies of synchronization in numerical models, such as the work by Pikovsky et al. (2001), Pecora et al. (1997), and Anishchenko et al. (1998), have shown that synchronization between two numerical systems (whether identical or not) can be achieved by adding a linear coupling term between the two systems. Experimental investigation have confirmed most of these numerical and theoretical approaches Wembe and Yamapi (1995); Yu et al. (1995). Several investigations have used this form of coupling to study synchronization in the classic and complex Lorenz equations (Pecora et al., 1997; Anishchenko et al., 1998; Pyragas, 1996; Rulkov et al., 1995; Stefanski et al., 1996), in the Rossler oscillator (Pecora et al., 1997; Osipov et al., 1997) and in other numerical systems (e.g. Guan et al., 1999; Boccaletti et al., 2002).

In a one-way coupling configuration this is performed by adding an extra term to the response system, proportional to the difference between the drive and response variables of the form:

$$
\begin{aligned}
& \frac{d x_{1}}{d t}=G\left(x_{1}\right), \\
& \frac{d x_{2}}{d t}=G\left(x_{2}\right)+\underbrace{\eta\left(x_{1}-x_{2}\right)}_{\text {Coupling }},
\end{aligned}
$$

where Eqs. (6a) and (6b) are the master and slave systems, respectively. $\eta$ is a parameter which determines the strength of the coupling. The choice of the $\mathbf{x}$ component that is used to couple the systems depends on the characteristics of the particular problem under investigation. It has been found that this type of interaction commonly leads to synchronization of periodic and chaotic oscillations as well as to chaos control.

Following these ideas, we coupled two 5-D two-layer models through a linear coupling term, perturbing directly the baroclinic mean flow correction term in the slave system. $X_{d}$ represents variations in the mean flow, which, as shown for the 5-D system, is associated with modulations of the amplitudes of the waves traveling in the channel. Thus our coupling term has the form $\eta\left(X_{d_{a}}-X_{d_{b}}\right)$, where $\eta$ is the coupling strength parameter. The complete one-way coupled, two-layer quasi-geostrophic model is then formed as follows

$$
\begin{aligned}
& \text { Master } \\
& \dot{A}_{s_{a}}=-\Delta_{s_{a}} A_{s_{a}}+\beta_{s_{a}} B_{s_{a}}-\left(v_{s_{a}}+\gamma_{s_{a}} X_{d_{a}}\right) B_{d_{a}}, \\
& \dot{B}_{s_{a}}=-\Delta_{s_{a}} B_{s_{a}}-\beta_{s_{a}} A_{s_{a}}+\left(v_{s_{a}}+\gamma_{s_{a}} X_{d_{a}}\right) A_{d_{a}}, \\
& \dot{A}_{d_{a}}=-\Delta_{d_{a}} A_{d_{a}}+\beta_{d_{a}} B_{d_{a}}-\left(v_{d_{a}}+\gamma_{d_{a}} X_{d_{a}}\right) B_{s_{a}}, \\
& \dot{B}_{d_{a}}=-\Delta_{d_{a}} B_{d_{a}}-\beta_{d_{a}} A_{d_{a}}+\left(v_{d_{a}}+\gamma_{d_{a}} X_{d_{a}}\right) A_{s_{a}}, \\
& \dot{X}_{d_{a}}=-\bar{\Delta}_{a} X_{d_{a}}+\overline{\gamma_{a}}\left[A_{s_{a}} B_{d_{a}}-B_{s_{a}} A_{d_{a}}\right],
\end{aligned}
$$$$
\begin{aligned}
& \text { Slave } \\
& \dot{A}_{s_{b}}=-\Delta_{s_{b}} A_{s_{b}}+\beta_{s_{b}} B_{s_{b}}-\left(v_{s_{b}}+\gamma_{s_{b}} X_{d_{b}}\right) B_{d_{b}}, \\
& \dot{B}_{s_{b}}=-\Delta_{s_{b}} B_{s_{b}}-\beta_{s_{b}} A_{s_{b}}+\left(v_{s_{b}}+\gamma_{s_{b}} X_{d_{b}}\right) A_{d_{b}}, \\
& \dot{A}_{d_{b}}=-\Delta_{d_{b}} A_{d_{b}}+\beta_{d_{b}} B_{d_{b}}-\left(v_{d_{b}}+\gamma_{d_{b}} X_{d_{b}}\right) B_{s_{b}}, \\
& \dot{B}_{d_{b}}=-\Delta_{d_{b}} B_{d_{b}}-\beta_{d_{b}} A_{d_{b}}+\left(v_{d_{b}}+\gamma_{d_{b}} X_{d_{b}}\right) A_{s_{b}}, \\
& \dot{X}_{d_{b}}=-\bar{\Delta}_{b} X_{d_{b}}+\bar{\gamma}_{b}\left[A_{s_{b}} B_{d_{b}}-B_{s_{b}} A_{d_{b}}\right]+\underbrace{\eta\left(X_{d_{a}}-X_{d_{b}}\right)}_{\text {Coupling }}
\end{aligned}
$$

In Eq. (7), as mentioned above, the coupling was introduced in the form of a linear term; which is a priori the simplest means of coupling two systems, and justified here since the precise details of how the coupling might occur in a real physical system are not yet characterized or fully understood. Note, however, that this term has the same form as Newton's classical law of cooling $d T / d t=K\left(T_{0}-T\right)$, which is appropriate for forced convective heat transfer. In experiments using the thermally driven rotating baroclinic annulus Hide and Mason (1975); Read et al. (1998) (which the single two-layer system attempts to model) the vertical shear in the velocity field is produced, in agreement with the thermal wind equation Andrews (2000), by a temperature gradient between the inner and outer wall of the annulus in a rotating frame of reference. This temperature difference is produced by nothing else but by forced convection between the wall and the fluid, since this is essentially how the thermal boundary conditions are maintained in typical baroclinic annulus experiments. The coupling term in the $X_{d}$ equation in our 5-dimensional formulation, therefore, effectively represents a zonally symmetric perturbation to the mean flow correction through Newton's law of cooling and the thermal wind equation. This represents a perturbation in the background thermal structure of the flow, associated, for instance, with a modulation of the temperature contrast between the inner and the outer cylinder, such as in the experiments carried out by Eccles et al. (2009).

The type of coupling where the interaction is only in one direction is usually termed master-slave. The first system acts as the master or driver, while the second one acts as the slave or driven system. In this configuration, the slave 
system is the one that will be perturbed, and it is the one that will be forced to follow the dynamics of the master. We have chosen to study this configuration first for one main reason: we have total control over the behaviour of the driver. The effects produced over the driven system can therefore be easily identified.

In order to study the synchronization between two nonidentical systems, a mismatch between the systems has to be introduced. Consequently, for the baroclinically unstable problem, the rotational Froude numbers, $F_{a, b}$, will be used to produce such a mismatch. Changes in the value of $F$ affect the parameters $\Delta_{d}, \beta_{d}, \bar{\Delta}, v_{d}, \bar{\gamma}$ and $\gamma_{d}$ of the model, and thereby produce shifts of the main oscillating frequency and its amplitude. In practice, in a real experiment, variations of $F$ would imply alterations in the thermal boundary conditions, in the aspect ratio of the annular container, or in the physical properties of the working fluid.

For the principal observable in each of our models, we have chosen $X_{d_{a, b}}$, the mean flow correction, since this is where the periodic and chaotic modulation of the amplitude of the baroclinic wave can be most easily identified, and can be seen as a direct extension of the modulated system by Eccles et al. (2006).

\section{Data analysis and detection of synchronized states}

There are different procedures to detect synchronization of periodic and chaotic oscillations. The most straightforward is via the classical Lissajous figure where the signal of one oscillator is plotted against the signal of the second. Regular patterns (even closed curves in the case of periodic dynamics) are usually an indication that synchronized behaviour could be developing. In chaotic systems, however, this test is less definitive.

A more useful and quantitative technique to detect synchronization in periodic and chaotic systems is the analysis of the phase difference between the external forcing and the oscillator or between the coupled oscillators. It has been proposed that the Hilbert transform is a suitable technique to calculate the instantaneous phase, even for reasonably smooth chaotic data (the Fourier spectrum of which contains a predominant main frequency or a narrow-band signal) (Pikovsky et al., 2001; Rosenblum and Pikovsky, 2003). In order to implement it, one has to construct from a scalar signal $s(t)$ a complex variable,

$\zeta=s(t)+i s_{H}(t)=A(t) e^{i \phi(t)}$,

where $s_{H}(t)$ is the Hilbert transform,

$s_{H}(t)=\pi^{-1} P \int_{-\infty}^{\infty} \frac{s(\tau)}{t-\tau} d \tau$.

In the above equation (Eq. 9), $\mathrm{P}$ denotes that the integral is taken in the sense of the Cauchy principal value. From this, the phase can be calculated using:

$\phi(t)=\arctan \frac{s_{H}(t)}{s(t)}$.

The phase obtained from Eq. (10) is restricted, by construction, to the interval $[0,2 \pi]$. When "unfolded" or "unwrapped" (i.e. by accumulating the phases such that every cycle, the phases $\phi_{1,2}$ increase by $2 \pi$ ) one can observe how it continuously grows in time, although some fluctuations will be observed, particularly for chaotic data. Finally, by calculating the phase difference between the oscillators, $\Delta \phi=\phi_{a}(t)-\phi_{b}(t)$ (where the subindex refers to the corresponding oscillator), we can determine whether the coupled system has reached a synchronized state or not. If the phase difference does not grow in time but remains bounded (i.e. it fluctuates around a constant value: $|\Delta \phi|<$ const) we have phase locking, generally understood as phase synchronization. Finally, we can create a histogram of $\Delta \phi \bmod (2 \pi)$ by subdividing the possible range of phase differences into $N$ intervals (of size $2 \pi / N$ and determine how often this difference occurs in each interval. In a phase synchronized state, this histogram will present a clear peak around a particular value of $\Delta \phi$. In contrast, in non-synchronized systems, this histogram is expected to be uniform.

Another condition for synchronization is based on the calculation of the frequency difference ir ratio. This condition is considered somewhat weak in investigations when the parameters of the systems are inaccessible ("passive" experiments (Pikovsky et al., 2001)) because the fact of having two interacting systems oscillating at (approximately) the same frequency does not imply that the two non-identical systems are necessarily phase locked; the closeness of the two frequencies could just be a coincidence. In our case, however, since we have full access an control over all the parameters (an "active" experiment), the computation of the frequency ratio will be a valuable tool in the detection of synchronized states. This will be especially helpful when analyzing the resulting dynamics when the mismatch and/or coupling strength can be varied over a relatively wide range of parameters Pikovsky et al. (2001).

To calculate the frequency of a chaotic signal, however, could be difficult. The most direct approach by calculating the differentiation of Eq. (10) could result in a fluctuating frequency $\Psi(t)$. This happens due to noise and the sometimes complicated form of the signal. Averaging over time can give much better values of the frequency

$\Psi=\left\langle\frac{d \phi(t)}{d t}\right\rangle$,

where \langle\rangle denotes averaging over time. This approximate mean frequency is usually usually known as the "observed frequency". By analysing its value, and in particular the difference and/or ratio between the frequencies of the two interacting systems, one can look for signatures of synchronization. 


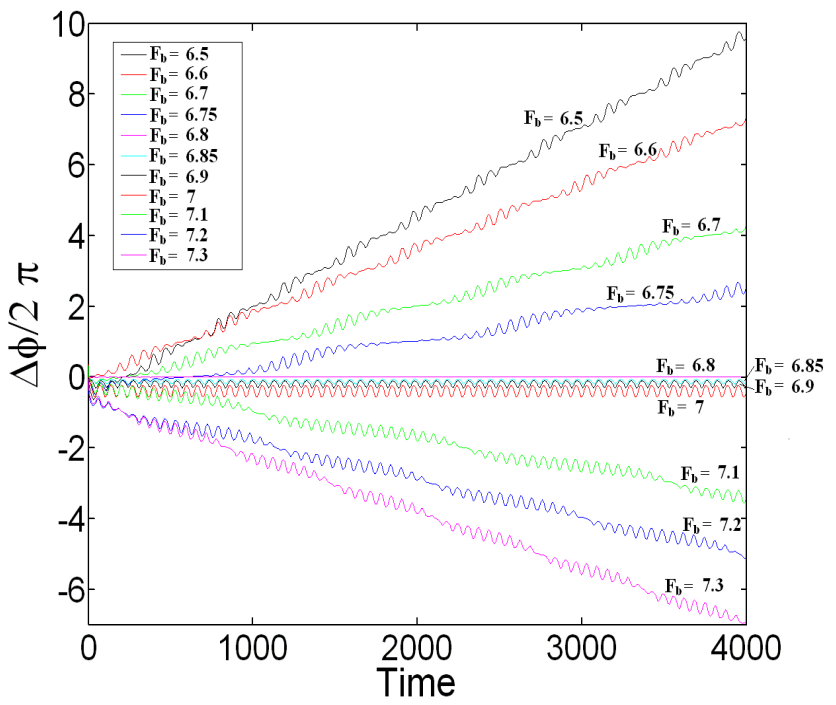

Fig. 1. Dynamics of $\Delta \phi$ at the synchronization transition. The phase difference is shown for several values of $F_{b}$ (which governs the frequency mismatch between the systems) for a constant value of the coupling strength $(\eta=0.002)$. In the synchronous state, for $F_{b}=6.8$ to 7 the plot show fluctuating but bounded $\Delta \phi$. Just outside the synchronized region the phase difference tends to grow in a smoothly stepped way $\left(F_{b}=6.7,6.75\right.$ and $\left.F_{b}=7.1\right)$ and more uniformly for larger mismatches $\left(F_{b}=6.5,6.6\right.$ and $\left.F_{b}=7.2,7.3\right)$. The transition at the other border of the synchronize regions occurs similarly, with difference that the phase difference now decreases in time. $F_{a}=6.8$ is always kept constant.

\section{Results}

First, we will describe the results of coupling the two systems when they are in a periodic regime (Sect. 5.1). Then we move on to perform a similar analysis in a chaotic regime (Sect. 5.2), where another tool to test for synchronization, namely the Auxiliary System Approach, was applied (Sect. 5.3).

\subsection{Periodic regime}

As a starting point, we will present the results of coupling the two systems in a periodic regime for various values of $F_{b}$ (with $F_{a}=6.8$ ) while keeping the coupling strength constant. Figure 1 shows extracts of the time variation of $\Delta \phi$ for various values of the mismatch (governed by changes in the value of the Froude number for the slave system, $F_{b}$ ) and a constant coupling parameter of $\eta=0.002$. For relatively large mismatches, the systems do not seem to synchronize, as expected. For values of $F_{b}$ between 6.7 and 6.75 however, the phase difference appears as an intermittent sequence of jumps (known as phase slips in this context) together with intervals of synchronized behaviour. These phase slips indicate that we are entering into the margins of a synchronized
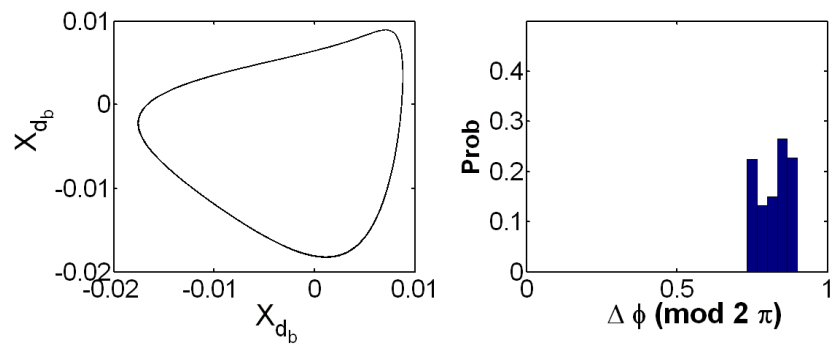

Fig. 2. Example of the Lissajous plot and the distribution of the cyclic relative phase, $\Delta \phi \bmod (2 \pi)$, for a synchronous state. $F_{b}=6.9 . F_{a}=6.8$ and $\eta=0.002$ as in Fig. 1 .

regime. When the mismatch is decreased, for the whole range from approximately $F_{b}=\sim 6.8$ to $\sim 7$, the phases of the two systems are synchronized; the phase difference in this state shows small fluctuations around a constant value. These fluctuations seem to increase in amplitude as the mismatch grows. When $F_{b}$ is larger than $\sim 7.05$, synchronization is lost and again a step-like change in the phase difference is observed. Figure 2 shows an example of the Lissajous plot and the distribution of the cyclic relative phase, $\Delta \phi \bmod (2 \pi)$, obtained when the systems are synchronized. A clear limit cycle in Fig. 2-(left) and a peaked distribution in Fig. 2-(right), confirm a phase synchronized state.

Figure 3, shows the bifurcation diagram of the slave system and the frequency ratio $\left(\Psi_{b} / \Psi_{a}\right)$ between the signals of the master and the slave system when the coupling strength is $\eta=0.002$. The region around $F_{b}=6.7$ to 7 , identified as a plateau in the frequency ratio plot, is evidently in a frequency entrained state. This is in complete agreement with what was found before and simply corroborates the findings plotted in Fig. 1. The regime diagram shows that the non-zero value of $\eta$ alters the amplitude of the oscillation of $X_{d_{b}}$. The bifurcation diagram does not, therefore, show a "thin line" (corresponding to a periodic behaviour) for the non-synchronized regions, but rather what appears at first sight to be chaotic behaviour. However, nonlinear time series analysis (Kantz and Schreiber, 1999, 2002) applied to the temporal signal of the slave in these regions showed that the dynamics in these regions is quasiperiodic and not chaotic. This dynamical state is characterized by having a multi-peaked frequency spectrum where different linear combinations of the two main periodic frequencies of each system are present.

In order to investigate synchronization using a wider range of parameters, a study where both $\eta$ and $F_{a}$ were varied continuously was carried out. In this case, we were looking for frequency entrainment between the two systems. We were therefore interested in ratio between the observed frequency of each system, $\Psi_{b} / \Psi_{a}$. The result of this investigation can be observed in the surface plot in Fig. 4. Along the horizontal axes we plot $F_{b}$, and $\eta$, and the frequency ratio, $\Psi_{b} / \Psi_{a}$ is plotted in the vertical, is shown in colour. $F_{a}$ is kept constant. 

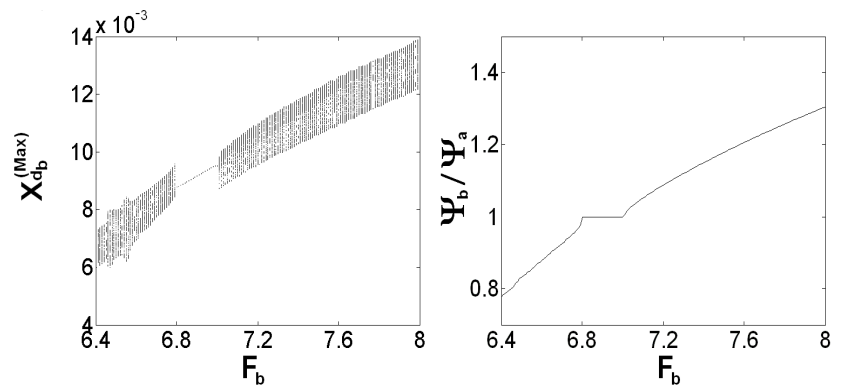

Fig. 3. (Left) Bifurcation diagram, constructed by acumulating the maxima of $X_{d_{b}}$ and plotting them against the varying control parameter $F_{b}$. In this case, the oscillation maxima are extracted from the $X_{d_{b}}(t)$ time series of the slave system after the transients have died out, and (Right) frequency difference for $F_{a}=6.8,6.4 \leq F_{b} \leq 8$, and $\eta=0.002$.

The regions where $\Psi_{b} / \Psi_{b}=1$ represent zones where a (1:1) frequency locked state exists. It is important to point out that the classic "Arnol'd tongue" behaviour (appearing as roughly triangular-shape synchronized regions in the $\eta-\Psi_{b}$ plane) found in many externally forced and master-slave systems was, to some extent, obtained at least for low values of $\eta$. Notice, however, that this tongue is not symmetrical and in fact is skewed to the right (towards larger $\Psi_{b}$ 's). This is essentially due to the fact that we are coupling two systems that change both frequency and amplitude when variations of the Froude number are applied, i.e. changes in $F$ will not only produce changes in the main oscillating frequency of the system, but also in its amplitude. This is in contrast to more classical external periodically-forced systems, where only changes in the frequency of the external forcing are typically applied, producing the well-known triangular synchronized regions in parameter space; known as Arnol'd tongues (Pikovsky et al., 2001). The result in our case is that the shape of the synchronized regions in the $(\eta-F)$ plane appears more irregular and complicated.

For larger values of the coupling strength, $(\eta>0.004)$, frequency entrainment is reached quite abruptly for $F_{b}$ smaller than $F_{a}$. On the other hand, for values of $\eta \approx 0.006$ and for $\Delta F \approx 0.8$ to 1.2 on the upper right hand side of the plot, we observe another phenomenon. Here, the frequency difference is not zero (as expected for a one-to-one frequency locking). Instead, we observe a ratio $\Psi_{b} / \Psi_{a}=2$. This means that the slave system is oscillating with a frequency which is double that of the master. The slave system suffered a bifurcation (frequency doubling) caused by the external perturbation. However, the system is still synchronized (but nowto a $1: 2$ frequency ratio).

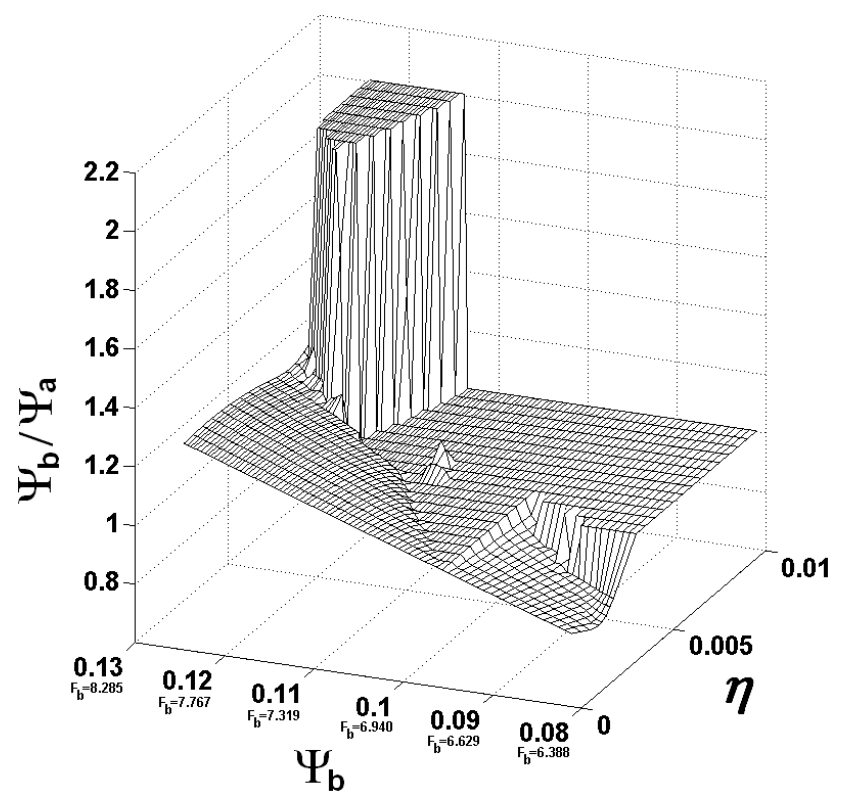

Fig. 4. Variations in the $\Psi_{b} / \Psi_{a}$ as a result of ramping $F_{b}$ from $F_{b}=6.4$ to 8 and $\eta$ from $\eta=0-0.01$ for a constant $F_{a}=6.8$ (which produces a $\left.\Psi_{b}=0.0956\right)$.

\subsection{Chaotic regime}

The dynamics obtained in the chaotic regime are equivalent to the modulated amplitude vacillation (MAV) flow regime found in baroclinic annulus experiments in the laboratory (Read et al., 1992) and numerical simulations (Read , 2003). MAV flows are typically characterized by having three or more independent frequencies and are, in some cases, found to show a chaotic behaviour. These vacillation regimes are primarily produced by nonlinear wave-mean flow interactions, though perhaps also modified in some circumstances by wave-wave interactions, as suggested in laboratory experiments in the rotating annulus (Hide and Mason, 1975; Read et al., 1998). The results for the coupled model in the chaotic regime follow more or less those found in the periodic regime. However, states such as imperfect phase synchronization (phase slips) are clearer.

Figure 5 shows a compilation of the phase difference $\Delta \phi$ for several values of the mismatch (governed by $F_{b}$ ) with a constant coupling strength $\eta$. There, it is possible to observe a clear transition to a synchronous state, starting with completely unsynchronized behaviour for a large mismatch $\left(F_{a}=13\right.$ and $\left.F_{b}=12\right)$, changing to imperfect phase synchronization (showing the characteristic phase slips) of various degrees, and finally to full phase synchronization, when the phase difference is bounded, i.e. the phase difference oscillates around a constant value (fulfilling the condition of phase synchronization according to Pikovsky et al., 2001; Rosemblum et al., 1996). 


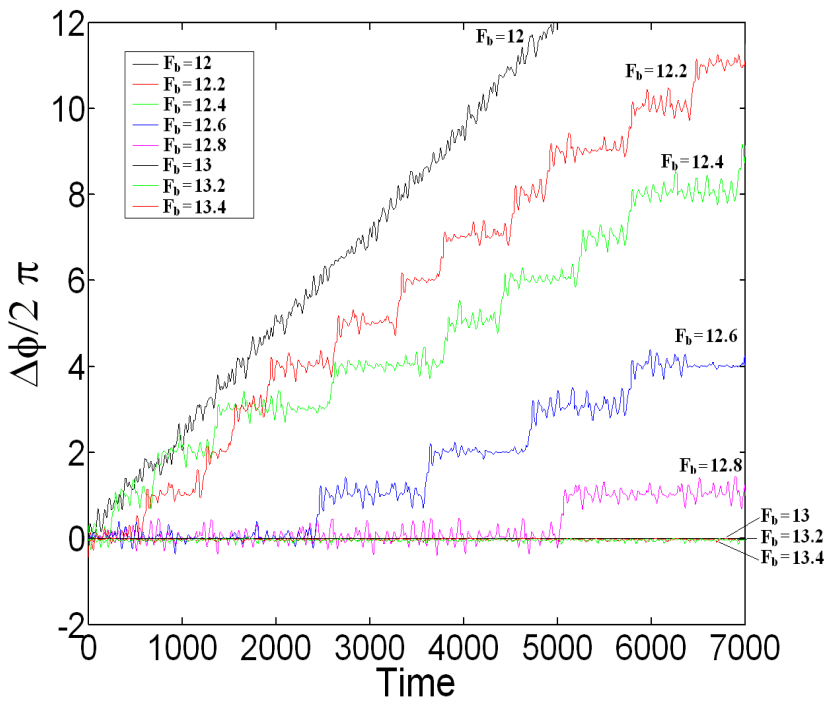

Fig. 5. Dynamics of $\Delta \phi$ at the synchronization transition. The phase difference is shown for several values of $F_{b}$ (which governs the frequency mismatch between the systems) for a constant value of the coupling strength $(\eta=0.05)$ and $F_{a}=13$. Imperfect phase synchronization is clearly visible for $F_{b}=12.2$ to $F_{b}=12.8$. As the detuning decreases, however, the number of phase slips decreases, demonstrating that the systems are entering into a synchronous behaviour. In the synchronous state, $\Delta \phi$ is bounded.
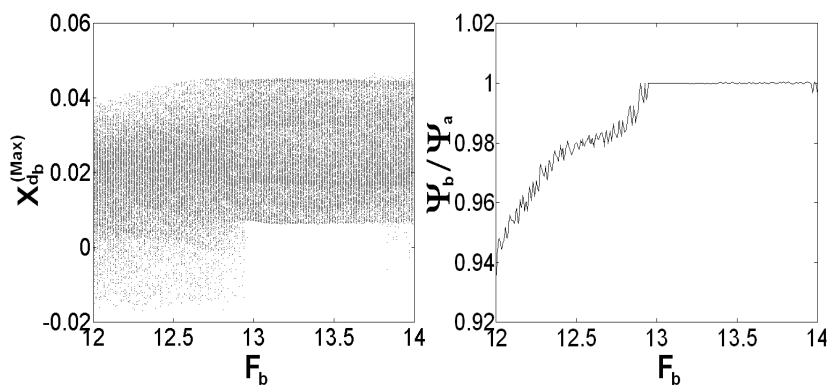

Fig. 6. Left: Bifurcation diagram in the chaotic case, and right: frequency difference for $F_{a}=13,12 \leq 14 \leq 8$, and $\eta=0.05$.

The phase difference found for $F_{b}=12$, shown in Fig. 5 tends to grow consistently with time, clearly indicating a lack of synchronization. This behaviour, however, changes radically when the mismatch is decreased. Signatures of phase synchronization start to develop, as is the case when values of $F_{b}=12.2$ to 12.8 are selected for the slave system. For $F_{b}>12.87$ and for the complete length of the run $\left(\sim 1 \times 10^{6}\right.$ timesteps) the dynamics of the slave were locked to those of the master system (as shown for the values of $F_{b}=13,13.2$ and 13.4.

Figure 6 shows the bifurcation diagram for the slave system and the frequency ratio for the whole range of $F_{b}$ stud-
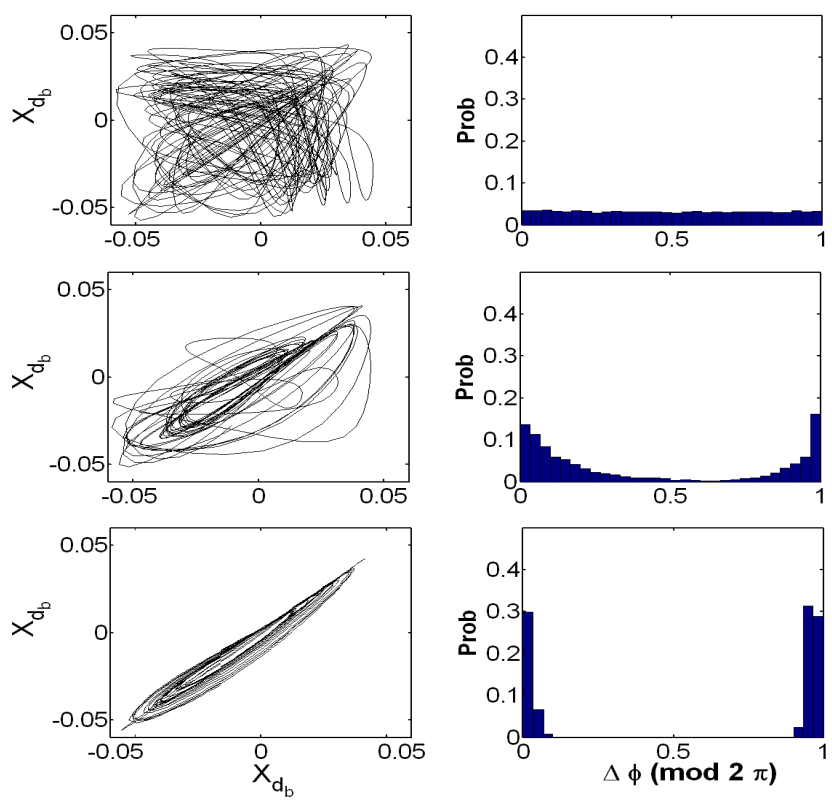

Fig. 7. Lissajous plot and the distribution of the cyclic relative phase, $\Delta \phi \bmod (2 \pi)$, for (top) unsynchronized case with $F_{b}=12$, (middle) imperfect phase synchronized with $F_{b}=12.6$, and (bottom) synchronized case with $F_{b}=13.2 . F_{a}=13$ and $\eta=0.05$ as in Fig. 6.

ied. It is possible to observe the synchronization transition at around $F_{b}=12.9$ in both plots. Three individual examples of an unsynchronized, a partially synchronized and a synchronized state, analyzed using the Lissajous plot and the distribution of the cyclic relative phase are presented in Fig. 7 . Notice the clear peak in the histogram for the last two cases, a clear signature of a preferred value in the phase difference even in the presence of spontaneous phase slips.

As performed in the periodic case, independently ramping the values of $\Psi_{b}$ (controlled by the value of $F_{b}$ ) and $\eta$ while computing the frequency ratio, $\Psi_{b} / \Psi_{a}$, can provide a map of the synchronized regions in the plane $\left(\Psi_{b}, \eta\right)$. The results of this analysis are presented in Fig. 8.

As can be seen in Fig. 8, a quite non-regular and asymmetric region of frequency entrainment is found (regions where $\left.\Psi_{b} / \Psi_{a} \approx 1\right)$. In particular the regions with $F_{b}<F_{a}$ need larger values of $\eta$ to synchronize probably due to the nonlinear response of the systems when $F$ (of either the master or slave systems) is varied. Variation in the Froude number not only induces variation in the observable oscillation frequency, but also in its amplitude. Therefore, the response of the slave system to the coupling is expected to be complex. Also, there is a region where, even though the two systems are set to evolve similarly by having very similar Froude numbers (where $\Delta F<0.2$ ), an increment in the value of the coupling strength is not directly reflected in an increase of the frequency entrainment. Furthermore, even for cases when 


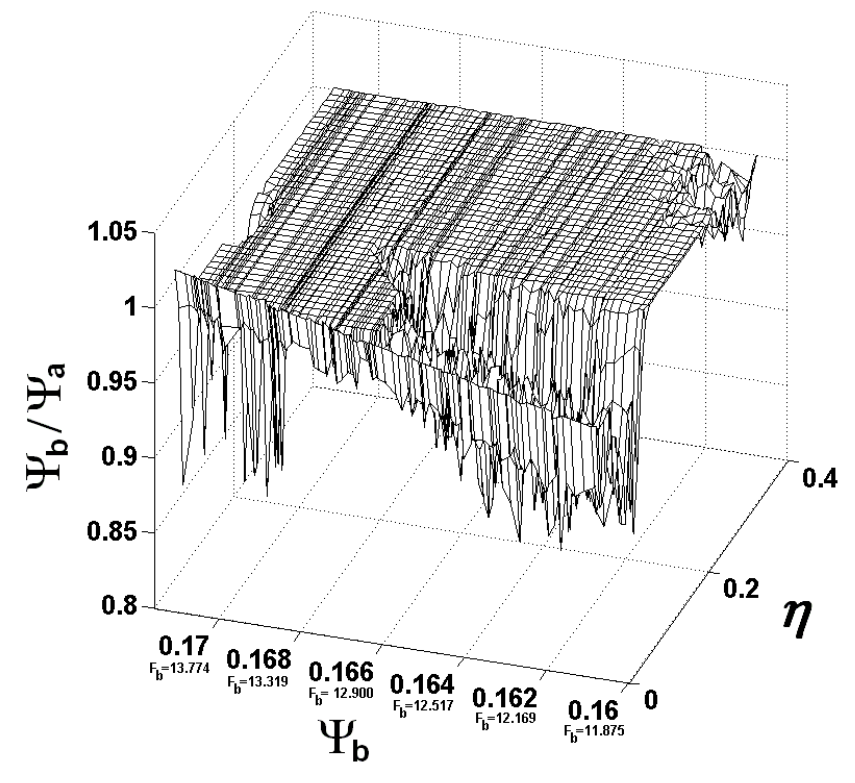

Fig. 8. The effects on the frequency ratio $\Psi_{b} / \Psi_{a}$ of ramping $F_{b}=12$ to $F_{b}=14$ and $\eta=0.0$ to $\eta=0.4$ whilst holding $F_{a}=13$ (which produces a $\Psi_{b}=0.1665$ ) in a MAV regime.

$\Delta F \simeq 0$ (i.e. $F_{a} \simeq F_{b}=13$ ) and $\eta=0.11$ to $\eta=0.12$, the two systems oscillate at slightly different (observable) frequencies, and only intermittently being partially locked, showing the typical phase slips characteristic of imperfect synchronized states.

\subsection{Generalized synchronization: the auxiliary system approach}

Synchronization of chaos is often understood as a phenomenon where two systems, in a chaotic regime, exhibit identical oscillations. However, in a master-slave configuration this regime of identical oscillations only occurs at certain points in the parameter space. Moreover, for nonidentical systems, strictly speaking that cannot happen. In particular, for a large coupling strength one could find a functional relationship between the states of two coupled systems. This means that we can equate dynamical variables from one system with a function of variables of another system. This type of regime is called generalized synchronization (Rulkov et al., 1995; Pikovsky et al., 2001). Using this definition, complete synchronization will be reached when the relations between the variables of the coupled systems are simple identity functions. Therefore, the presence of generalized synchronization between slave $\mathbf{x}_{s}(t)$ and master $\mathbf{x}_{m}(t)$ chaotic systems means that there is a functional relation of the type $\mathbf{x}_{s}(t)=\mathbf{F}\left[\mathbf{x}_{m}(t)\right]$ between the systems, after the transients have decayed away.

In this work, we have used the auxiliary system approach as another alternative method to study synchronization, in the generalized sense, in our coupled system. A detailed formulation of the technique is presented by Rulkov et al. (1995), and some examples of its use are presented in the work by Hramov et al. (2005) and Hramov and Koronovskii (2005b). In that technique, we consider the dynamics of the master $\mathbf{x}_{m}(t)$ and the slave $\mathbf{x}_{s}(t)$ systems. We also introduce an auxiliary system $\mathbf{x}_{a s}(t)$ which is identical to the slave system (it is important to note that the coupling to the master is also exactly the same) but that starts with slightly different initial conditions (i.e. $\mathbf{x}_{s}\left(t_{0}\right) \neq \mathbf{x}_{a s}\left(t_{0}\right)$ ). In the absence of generalized synchronization, the trajectories of the two systems in phase space will share the same attractor but will move apart rapidly until their separation is of the order of the attractor, and they will then remain uncorrelated as they develop. However, if the two response systems (slave and auxiliary) are synchronized to the master through the generalized synchronization relation $\mathbf{x}_{s}(t)=\mathbf{F}\left[\mathbf{x}_{m}(t)\right]$ and $\mathbf{x}_{a s}(t)=\mathbf{F}\left[\mathbf{x}_{m}(t)\right]$, then it is clear that (after transients die away) a solution with the form $\mathbf{x}_{s}(t)=\mathbf{x}_{a s}(t)$ exists. This technique allows a relatively easy way to identify generalized synchronization of chaotic systems by comparing the signal of two identical response systems, i.e. one needs to look for the identity $\mathbf{x}_{s}(t)=\mathbf{x}_{a s}(t)$.

Therefore, in order to detect generalized synchronization in the coupled two-layer system:

1. an auxiliary system which is identical to the slave system, coupled to the master by a coupling term identical to the one used in the slave system, was constructed. The only difference is that the new system (auxiliary) is initialized with slightly different initial conditions.

2. the signals from the two response systems (slave and auxiliary, $X_{d_{b}}$ and $X_{d_{a s}}$ ) were compared, either by plotting the corresponding Lissajous figures (master vs. slave, master vs. auxiliary, and slave vs. auxiliary), by looking for the $X_{d_{b}}=X_{d_{a s}}$ condition to be satisfied, or by means of simple linear correlation, in this case looking only at the mean flow correction term.

As the technique suggests, one has to ensure that any transient has died away before starting to look for synchronization and to compare the signals from the two slave systems. Several time spans were tried, therefore, and 1000 timesteps was generally found to be long enough for the transients to decay. To ensure that this condition was satisfied, however, the running time was set to 100000 timesteps.

In Figs. 9, 11 and 13, extracts from the time series, and Lissajous plots for different numerical runs are shown. Phase differences and plots of $X_{d_{b}}-X_{d_{a s}}$ are presented in Figs. 10, 12 and 14.

In Fig. 9, with $\eta=0$, no synchronization is expected. The two response (slave) systems, having been initialized with different initial conditions and being chaotic, evolve differently. This is also corroborated by plotting the phase difference between the master and the slave systems and between 

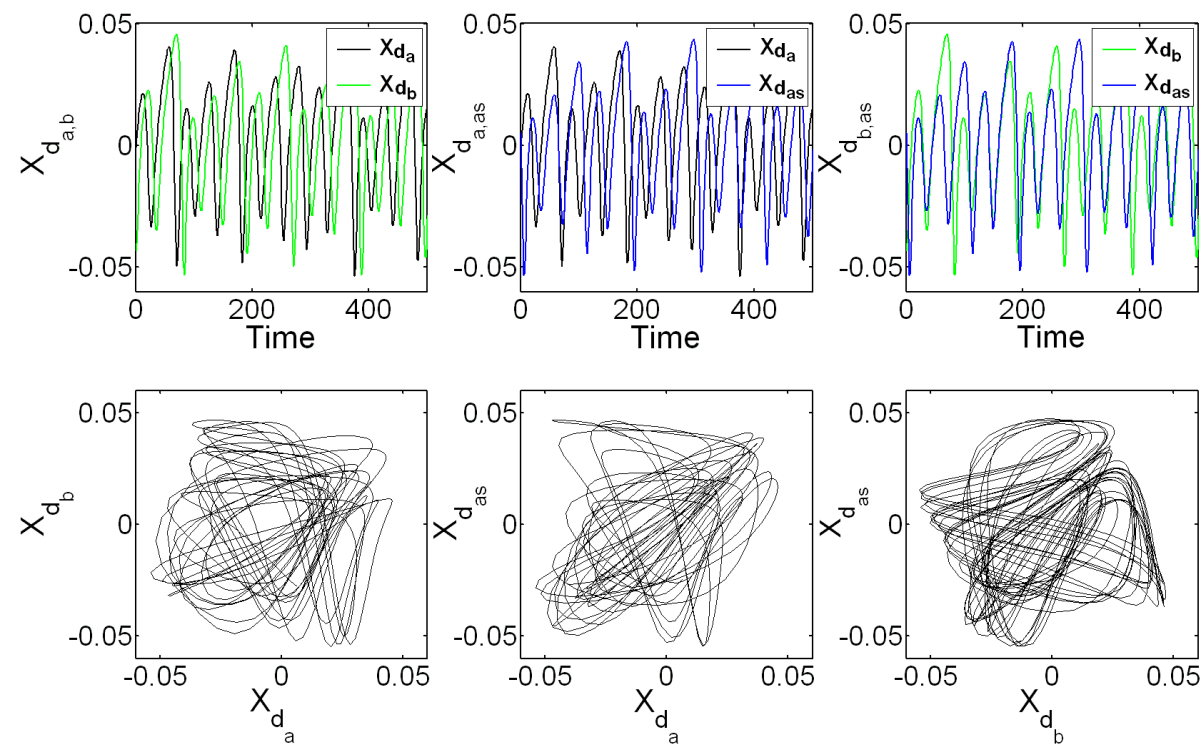

Fig. 9. Results of the auxiliary system approach applied to the system with $F_{a}=13, F_{b}=12.6$ and $\eta=0$. The top row shows (from left to right) extracts of the time series of the mean flow correction term of the master, $X_{d_{a}}$, (in black) and the first slave $X_{d_{b}}$ (in green); the master (in black) and the auxiliary system $X_{d_{a s}}$ (in blue); and the two response systems, respectively. Projections onto the planes $\left(X_{d_{a}}, X_{d_{b}}\right.$ ), $\left(X_{d_{a}}, X_{d_{a s}}\right),\left(X_{d_{b}}, X_{d_{a s}}\right)$ are shown in the bottom row.

the master and the auxiliary systems, respectively, as presented in Fig. 10. This plot shows that the phase of the two response systems is not bounded, and they evolve differently. In the same way, the projections into the planes $\left(X_{d_{a}}, X_{d_{b}}\right)$, $\left(X_{d_{a}}, X_{d_{a s}}\right)$ and $\left(X_{d_{b}}, X_{d_{a s}}\right)$ show no correlation between the systems, not even between the two response systems.

For the case with $\eta=0.066$, we can observe phase slips in the two response systems. It is important to note that the phase slips occur at different times for each system, as can be seen in Figs. 11 and 12.

A further increment in the value of the coupling strength will lead the systems to a phase synchronized state. The two response systems are phase synchronized to the common master, as can be seen in Figs. 13 and 14. However, even though the coupled systems are in a phase synchronized state, they are not yet synchronized in the generalized sense. Figure 14, which shows that the signals of the two response systems are not identical. The condition for generalized synchronization via the auxiliary system approach is not yet fulfilled.

For $\eta>0.2$, generalized synchronization is finally found. Figures 15 and 16 show this state. The Lissajous plot in the planes $\left(X_{d_{a}}, X_{d_{b}}\right),\left(X_{d_{a}}, X_{d_{a s}}\right)$ show that the oscillation of the drive and response systems are not identical; however, the two response systems are phase synchronized to the master (Fig. 16 shows that the phase difference is bounded) and their time series are identical. This can be corroborated by looking at the projection of the trajectories onto $\left(X_{d_{b}}, X_{d_{a s}}\right)$ plane, which is basically a line. $X_{d_{b}}(t)-X_{d_{a s}(t)}$ (shown in Fig. 16-
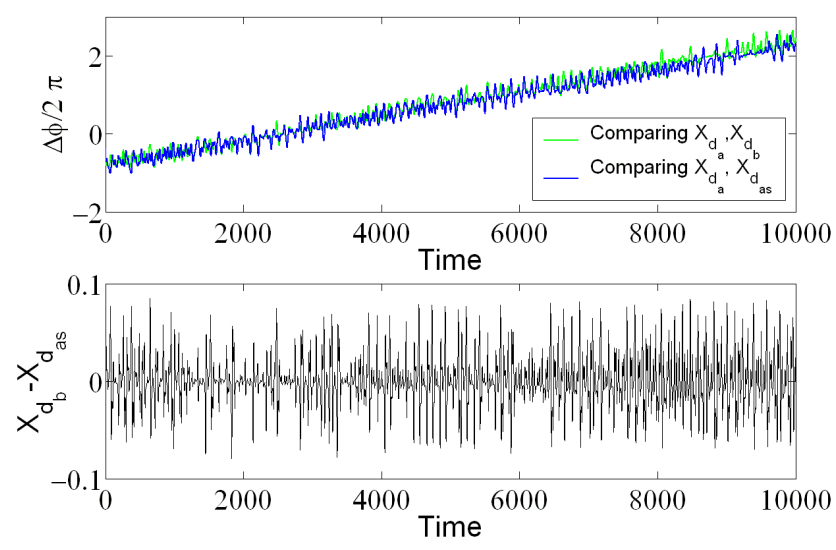

Fig. 10. Top: Phase difference between the master and slave (in green) systems and between the master and Auxiliary systems (in blue), calculated from the data presented in Fig. 9. Notice the lack of any phase locking between the systems. Bottom: The dependence of the difference between the coordinates of the slave and auxiliary systems.

bottom) is equal to zero which shows that the oscillations are identical for the whole running time, fulfilling the condition for generalized synchronization. Therefore these systems are synchronized in the generalized sense.

We can then apply this analysis for the same range of parameters used to study frequency entrainment presented in Fig. 8. The result of this is shown in Fig. 17. On the 

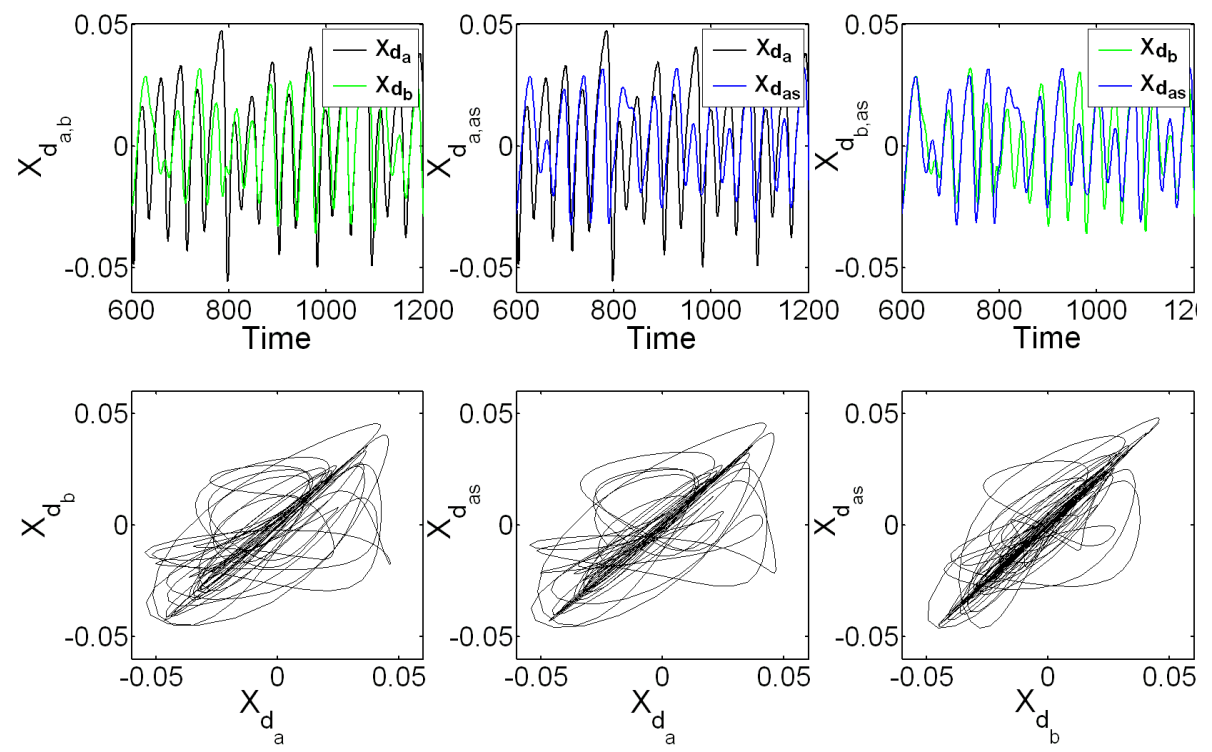

Fig. 11. As for Fig. 9 but with a coupling strength of $\eta=0.066$. A clear phase slip can be noticed in $X_{d_{a s}}$ at Time $\approx 820$.
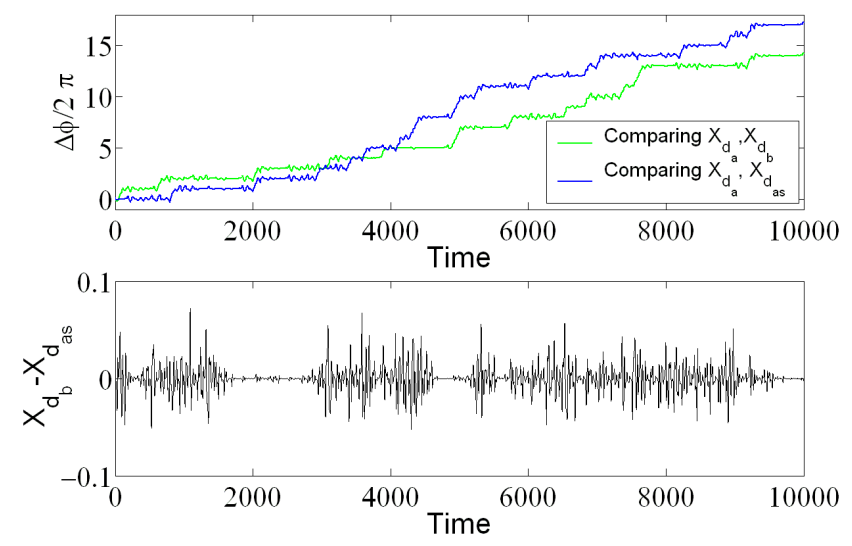

Fig. 12. Top: Phase difference between the master and slave (in green) systems and between the master and Auxiliary systems (in blue), calculated from the data presented in Fig. 11. Notice that the phase jumps or phase slips are quite random and that they occur at different times in each system. Bottom: The dependence of the difference between the coordinates of the slave and auxiliary systems. Short epochs of correlated amplitude can be observed.

horizontal axis we have $F_{b}$ (keeping $F_{a}=13$ while varying the value of the response system's Froude number from $F_{b}=12$ to $F_{b}=14$ ), in the vertical the value of the coupling strength, $\eta$, and in colours the value of the linear correlation coefficient between the two response systems. A correlation approximately equal to 1 is used to determine when the two response systems are identical and therefore that the systems are synchronized in a generalized sense.
As can be noticed, when compared with the results shown in Fig. 8, the regions of synchronized behaviour in the two plots are alike. Larger values of $\eta$, however, are generally needed, in agreement with the literature, to reach a generalized synchronized state.

During the analysis, particularly near the boundaries between phase synchronized and generalized synchronized regions, a state which is called by some authors intermittent generalized synchronization (Hramov and Koronovskii, 2005b), was also found. In this state, the two response systems (slave and auxiliary) are phase synchronized with the common master. However, the distinctive feature in this particular region is that the oscillations of the two response systems are identical for short periods of time, then become unrelated for quite a few timesteps, and then related again and so on in a random way. It is interesting to point out that the amplitude of these irregular "bursts" (in the $X_{d_{b}}-X_{d_{a s}}$ plot) is comparable to the amplitude of the natural oscillation of the unperturbed systems. An example of this behaviour is shown in Fig. 18. It is recommended that in future work, some further attention should be put into this particular synchronized state, and a more detailed analysis should be performed.

\section{Discussion and final remarks}

In this paper, results of numerical experiments performed using a coupled system of a pair of two-layer quasigeostrophic models of baroclinic instability were presented. We found that the implementation of the coupling, applied solely through the mean flow correction term, not only follows the 

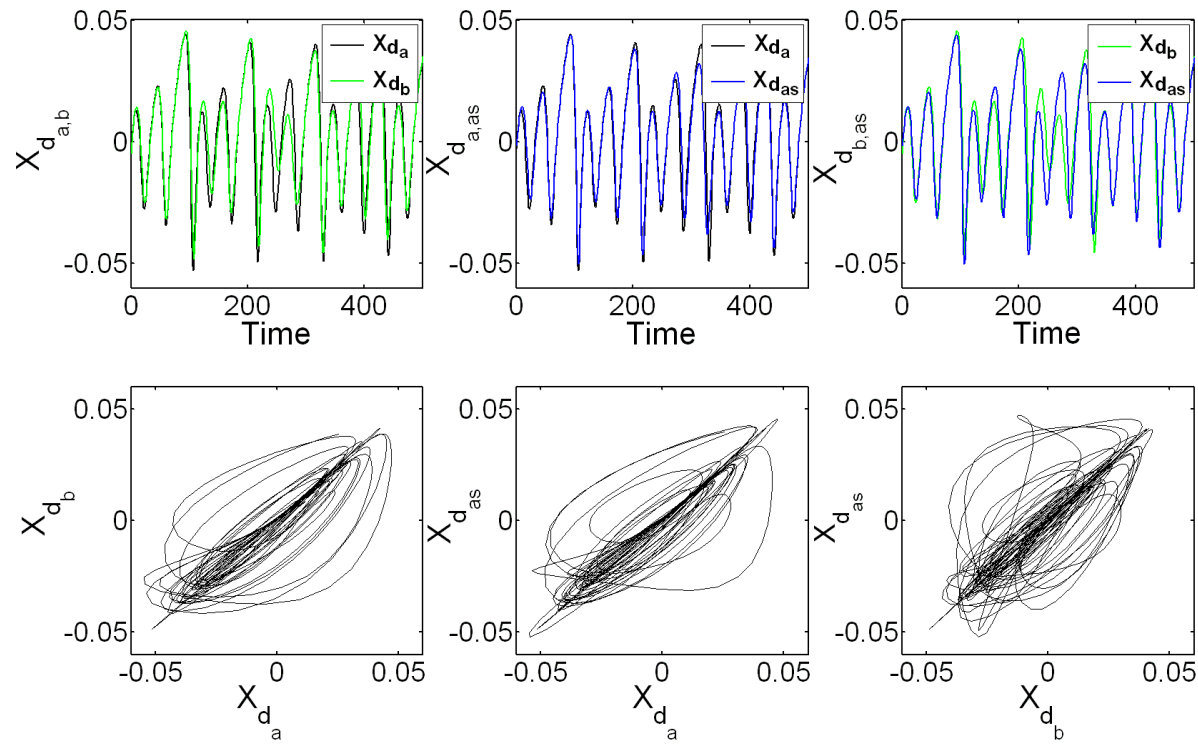

Fig. 13. As for Fig. 9 but with a coupling strength of $\eta=0.155$. Note that the two response systems are phase synchronized (to the common master). It is still visible, however, that the two response systems are not identical.

pattern proposed in various previous investigations, but it also has a physical interpretation in association with an experimental counterpart, the thermally driven baroclinic annulus. We studied the effects of this coupling in the periodic (Amplitude Vacillation, AV) and chaotic (Modulated Amplitude Vacillation, MAV) regimes previously identified and reported in Lovegrove et al. (2001) and Lovegrove et al. (2002). Nonlinear time series analysis, phase dynamics, and bifurcation diagrams were used to study the dynamics of the coupled system. Depending upon the strength of coupling, the mismatch and the type of dynamics of the systems (either in a periodic or a chaotic regime), various degrees of synchronization were found such as imperfect synchronization, phase synchronization, generalized synchronization and intermittent generalized synchronization were found. It was also found that the synchronization regions in the $(\eta, F)$ plane follow an Arnol'd tongue-like behaviour. However, the shape is not entirely triangular (as in the classic view of an Arnol'd tongue in externally forced systems). This was expected due to the complex dependence of both the frequencies and the amplitudes of the oscillation on changes in the Froude number and also due to the large coupling strengths used in the runs. The auxiliary system approach was used to seek for generalized synchronization in the chaotic regime. This proved to be a valuable technique to analyse and study another degree of synchronized states. The synchronization regions found with both the frequency-ratio diagnostic (Fig. 8) alone and via the auxiliary system approach (Fig. 17) gave similar results regarding the general shape of these regions. It was found, however, that larger values of $\eta$ are needed to cross the boundary from phase synchronized states
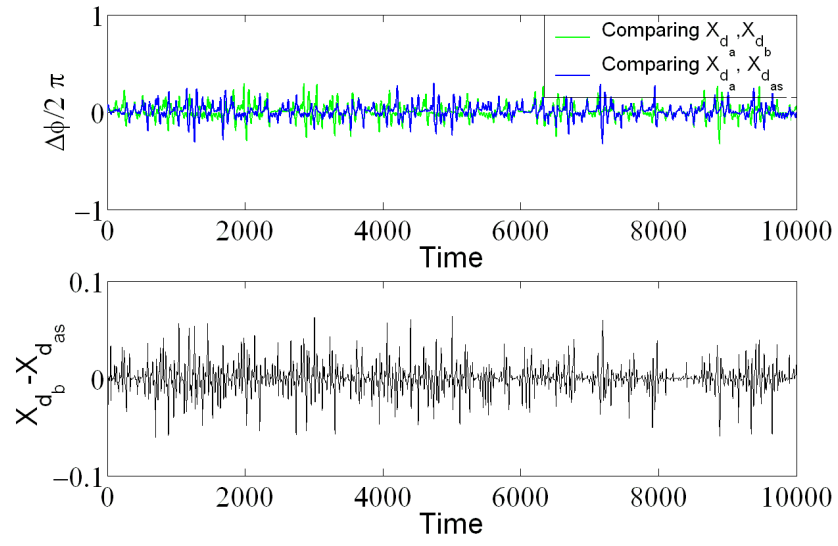

Fig. 14. Top: Phase difference between the master and slave systems (in green) and between the master and Auxiliary systems (in blue), calculated from the data presented in Fig. 13. Notice that the two response are phase locked to the master. Although the phase difference presents small fluctuations, it is bounded. Bottom: Plot of $X_{d_{a s}}-X_{d_{b}}$. Even though the two response systems are phase synchronized, there is yet not full correlation between their amplitudes and therefore, the conditions of generalized synchronization is not fulfilled.

to generalized synchronized states. In general, it was also found that relatively larger coupling strengths (for similar mismatches) were needed to reach phase synchronization in chaotic regimes than in periodic ones.

Due to its simplicity, it is not easy to directly apply our findings and results to the explanation of real atmospheric 

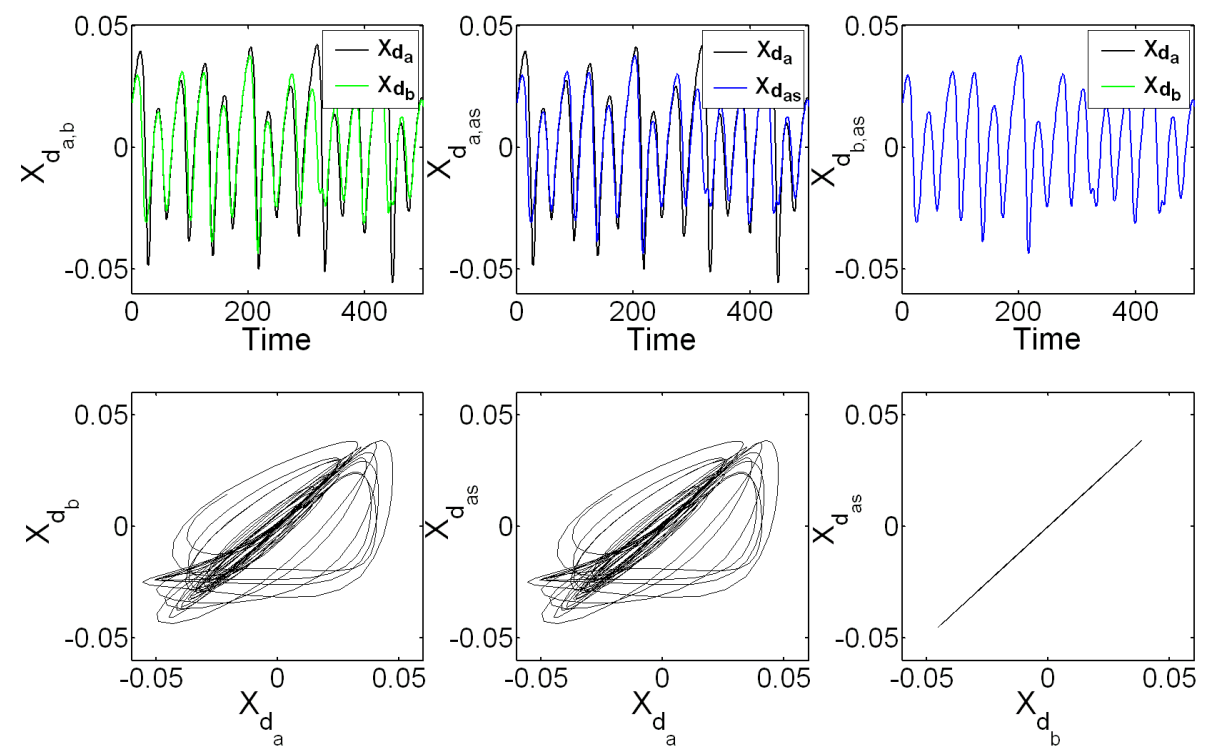

Fig. 15. As for Fig. 9 but with a coupling strength of $\eta=0.2$. Generalized synchronization if finally reached. Notice the perfect correlation between the amplitudes of the two response systems.
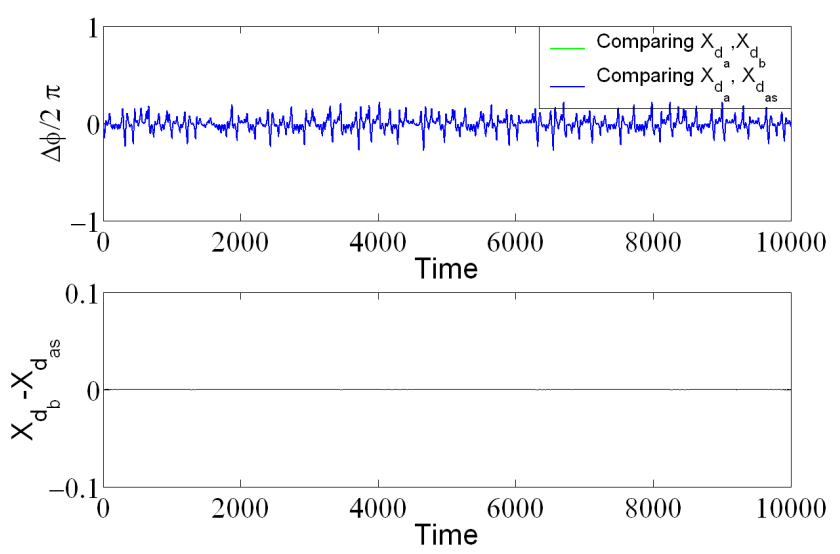

Fig. 16. Top: Phase difference between the master and slave (in green) systems and between the master and Auxiliary systems (in blue) calculated from the data presented in Fig. 15. Notice that the phase difference between the response systems and the master is the same. In fact, it is not possible to observe the blue line as it is completely shadowed by the green one. Bottom: The dependence of the difference between the coordinates of the slave and auxiliary systems on time for the data shown in Fig. 15.

synchronized and highly correlated phenomena. Our numerical experiments, however, can qualitatively illustrate some of the possible behaviour expected in both, possible real experiments in the laboratory, or particular and specific pairs of quasi-unidirectionally coupled geophysical phenomena such as the cases presented by Maraun and Kurths (2005). The possibility that synchronization, and the coupling that leads

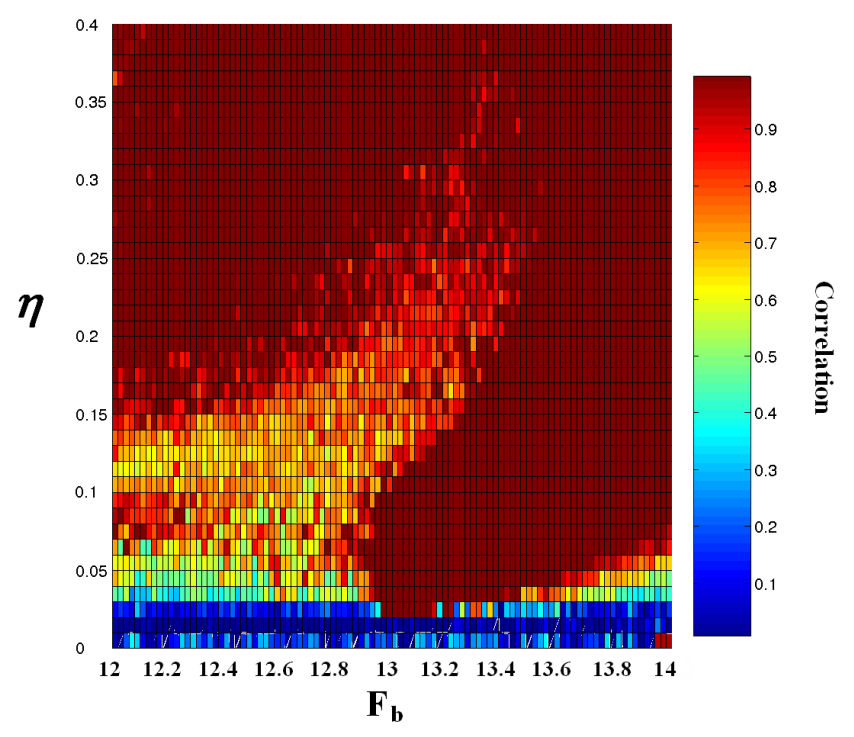

Fig. 17. Generalized synchronization regime diagram. Results of the correlation between the two response systems for different values of $F_{b}$ and $\eta ; F_{a}=13$. Correlation $\approx 1$ means that the two response systems are oscillating identically and therefore we reached a synchronized state in the generalized sense between the oscillations in the amplitude of the two baroclinic waves.

to associated phase-locking, could itself vary with time is another factor that is only just beginning to receive attention. But recent studies have indicated that the onset of synchronization between disparate climate signals can be stimulated by discrete events, such as a major volcanic eruption Maraun and Kurths (2005). 

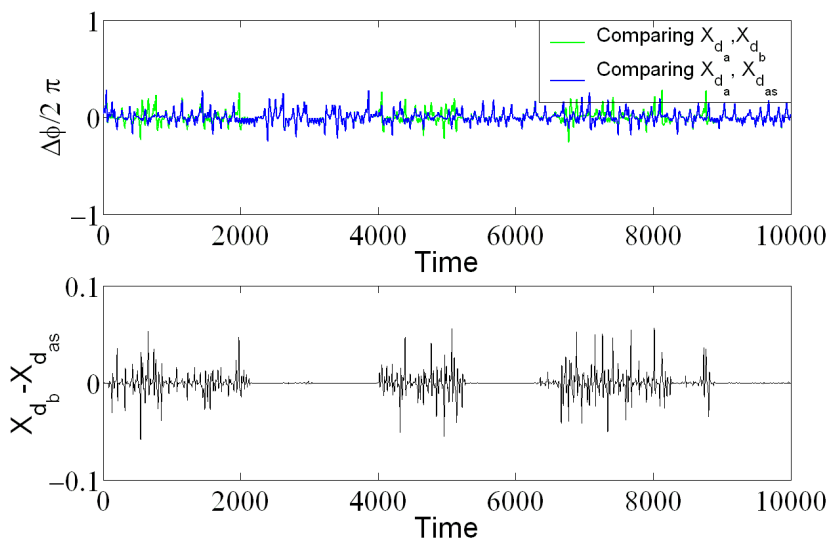

Fig. 18. Top: The difference of phase between the master and the two respose systems. Bottom: The dependance of the difference between the coordinates of the slave and auxiliary systems on time. $F_{a}=13, F_{b}=12.6$ and $\eta=0.162$.

The correlated atmospheric phenomena found by Duane et al. (1999) could, therefore, be an atmospheric manifestation of synchronization of two coupled extended systems. This represents an unusual, and poorly understood, type of teleconnection that has been identified recently and that is not easily explained in terms of linear dynamics. The blocked states essentially represent a particular phase in the respective zonal index cycles of the two hemispheres, as introduced in the first sections of this paper. However, the mechanism for producing such correlated behaviour between two hemispheres, often at quite different points in their respective seasonal cycles, is far from clear. The main aim of our paper is to look for some insight into the significance of studying synchronization in a geophysically-relevant simple nonlinear model. Traditionally, the two layer model has been used as a test scenario for the understanding of mid-latitude generation of baroclinic waves. In this same spirit and inspired by the findings presented in Duane et al. (1999); Duane and Tribbia (2001), in its most direct form, our coupled system models the interaction between two interconnected middlelatitude baroclinic channels, where each two-layer system represents one hemispheric channel. In a system as complex as the Earth, the complicated, intermittent and nonlinear interactions between sub-systems represent enormous challenges to the modelling community as it tries to emulate the final state of these interactions accurately and realistically. In this context, a knowledge and understanding of generic interactions between dynamical systems in the presence of nonlinearity is vital to guide the future development of modelling strategies. Until recently, the approach of the climate science community has tended to focus upon methods based on linearised theory and statistical analysis to guide the formulation of modelling and theoretical interpretations of observed phenomena. However, methodologies deriving from nonlinear systems are beginning to be more widely recognized and applied, amongst which are those related to chaos synchronization in coupled systems and networks.

One of the main improvements from the periodic forced system presented in Eccles et al. (2006) is that we are able to perturb a system, the slave system, with chaotic signals, bringing it closer to a possible real situation, since forcing in the atmosphere (and even in well controlled laboratory experiments) are never usually monochromatic or periodic, but rather chaotic and noisy.

Acknowledgements. This work supported by the UK Natural Environment Research Council via a Dorothy Hodgkin award and grant ref. NE/F002157/1.

Edited by: U. Feudel

Reviewed by: two anonymous referees

\section{References}

Andrews, D.: An Introduction to Atmospheric Physics, Cambridge University Press, pp. 140-153, 2000.

Anishchenko, V. S., Silchenko, A. N., and Khovanov, I. A.: Synchronization of switching processes in coupled Lorenz systems, Phys. Rev. E, 57(1), 316-322, 1998.

Appleby, J.: Comparative theoretical and experimental studies of baroclinic waves in a two-layer system, $\mathrm{PhD}$ Thesis, University of Leeds, UK, 1982.

Boccaletti, S., Kurths, J., Osipov, G., Valladares, D. K., and Zhou, C. S.: The synchronization of chaotic systems, Phys. Rep., 366, 1-101, 2002.

Duane, G. S.: Synchronized chaos in extended systems and meteorological teleconnections, Phys. Rev. E, 56, 4298-4301, 1997.

Duane, G. S., Webster, P. J., and Weiss, J. B.: Co-occurrence of Northern and Southern Hemisphere Blocks as Partially Synchronized Chaos, J. Atmos. Sci., 56, 4183-4205, 1999.

Duane, G. S. and Tribbia, J. J.: Synchronized chaos in Geophysical Fluid Dynamics, Phys. Rev. Lett., 86, 6475-6491, 2001.

Eccles, F. J. R., Read, P. L., and Haine, T. W. N.: Synchronization and chaos control in a periodically forced quasi-geostrophic two-layer model of baroclinic instability, Nonlin. Processes Geophys., 13, 23-39, 2006, http://www.nonlin-processes-geophys.net/13/23/2006/.

Eccles, F., Read, P. L., Castrejon-Pita, A. A., and Haine, T. W. N.: Synchronization of modulated traveling baroclinic waves in a periodically forced, rotating fluid annulus. Phys. Rev. E, 79, 015202R-1 015202R-4, 2009.

Früh, W.-G. and Read, P. L.: Wave interactions and the transition to chaos of baroclinic waves in a thermally-driven, rotating cylindrical annulus, Philos. T. R. Soc. Lond., A355, 101-153, 1997.

Gildor, H. and Tziperman, E.: Sea ice as the glacial cycles' climate switch: Role of seasonal and orbital forcing, Paleoceanography, 15(6), 605-615, 2000.

Guan, S., Lai, C. H., and Wei, G. W.: Phase synchronization between two essentially different chaotic systems, Phy. Rev. E, 72, 016205-3-016205-8, 2005.

Hart, J. E.: Finite-amplitude baroclinic instability with periodic forcing, Physica D, 39, 239-261, 1989. 
Hide, R. and Mason, P. J.:. Sloping convection in a rotating fluid, Adv. Phys., 24, 47-100, 1975.

Holton, J. R.: An Introduction to Dynamic Meteorology, Elsevier Science and Technology, UK, 4th edn., 2004.

Hramov, A. E., Koronovskii, A. A., and Popov, P. V.: Generalized synchronization in coupled Ginzburg-Landau equations and mechanisms of its arising, Phys. Rev. E, 72, 037201-1-4, 2000.

Hramov, A. E. and Koronovskii, A. A.: Intermittent generalized synchronization in unidirectionally coupled chaotic oscillators, Europhys. Lett., 70(2), 169-175, 2005.

Hegger, R., Kantz, H., and Schreiber, T.: Practical implementation of nonlinear time series methods: The TISEAN package, Chaos, 9, 413-435, 1999.

Kantz, H. and Schreiber, T.: Nonlinear time series analysis, Cambridge University Press, Cambridge UK, 2002.

Lovegrove, A.: Bifurcations and instabilities in rotating two-layer fluids, PhD thesis, University of Oxford, UK, 1997.

Lovegrove, A., Moroz, I., and Lovegrove, A. F., Moroz, I. M., and Read, P. L.: Bifurcations and instabilities in rotating two-layer fluids: I.f-plane, Nonlin. Processes Geophys., 8, 21-36, 2001, http://www.nonlin-processes-geophys.net/8/21/2001/.

Lovegrove, A., Lovegrove, A. F., Moroz, I. M., and Read, P. L.: Bifurcations and instabilities in rotating, two-layer fluids: II. $\beta$ plane, Nonlin. Processes Geophys., 9, 289-309, 2002, http://www.nonlin-processes-geophys.net/9/289/2002/.

Lunkeit, F.: Synchronization experiments with an atmospheric global circulation model, Chaos, 11, 47-51, 2001.

Maraun, D. and Kurths, J.: Epochs of phase coherence between El Niño/Southern Oscillation and Indian Monsoon, Geophys. Res. Lett., 32, L15709, doi:10.1029/2005GL023225, 2005.

Osipov, G. V., Pikovsky, A. S., Rosenblum, M. G., and Kurths, J.: Phase synchronization effects in a lattice of nonidentical Rössler oscillators, Phys. Rev. E, 55, 2353-2361, 1997.

Pecora, L. M. and Carrol, T. L.: Synchronization in chaotic systems, Phys. Rev. Lett., 68(8), 821-824, 1990.

Pecora, L. M., Carroll, T. L., Johnson, G. A., and Mar, D. J.: Fundamentals of synchronization in chaotic systems, concepts and applications, Chaos, 7, 520-543, 1997.

Pedlosky, J.: Finite-Amplitude Baroclinic waves, J. Atmos. Sci., 27, 15-30, 1970.

Pedlosky, J.: Finite-Amplitude Baroclinic waves with small dissipation, J. Atmos. Sci., 28, 587-597, 1971.

Pedlosky, J.: Limit cycles and unstable baroclinc waves, J. Atmos. Sci., 29, 53-63, 1972.
Phillips, N. A.: Energy transformations and meridional cirulations associated with simple baroclinic waves in a two-level quasigeostrophil model, Tellus, 6, 273-286, 1954.

Pikovsky, A., Rosenblum, M., and Kurths, J.: Synchronization: A universal concept in nonlinear sciences, Cambridge University Press, Cambridge, UK, 2001.

Pyragas, K.: Weak and strong synchronization of chaos, Phys. Rev. E, 54(4), R4508-R4511, 1996.

Read, P. L.: A combined laboratory and numerical study of heat transport by baroclinic eddies, J. Fluid Mech., 489, 301-323, 2003.

Read, P. L., Bell, M. J., Johnson, D. W., and Small, R. M.: Quasiperiodic and chaotic flow regimes in a thermally driven, rotating fluid annulus, J. Fluid Mech., 238, 599-632, 1992.

Read, P. L., Collins, M., Früh, W.-G., Lewis, S. R., and Lovegrove, A. F.: Wave Interactions and Baroclinic Chaos: A Paradigm for Long Timescale Variability in planetary atmospheres, Chaos Soliton Fract., 9, 231-249, 1998.

Rosenblum, M. and Pikovsky, A.: Synchronization: from pendulum clocks to chaotic lasers and chemical oscillators, Contemp. Phys., 44, 401-416, 2003.

Rosenblum, M., Pikovsky, A., and Kurths, J.: Phase synchronization of chaotic oscillators, Phys. Rev. Lett., 76, 1804-1807, 1996.

Rybski, D., Havlin, S., Bunde, A.: Phase synchronization in temperature and precipitation records, Physica A, 320, 601-610, 2003.

Rulkov, N. F., Sushchik, M. M., Tsimring, L. S., and Abarbanel, H. D. I.: Generalized synchronization of chaos in directionally coupled chaotic systems, Phys. Rev. E, 51, 980-994, 1995.

Salmon, R.: Lectures on geophysical fluid dynamics, Oxford University Press, Oxford, UK, 1998.

Stefanski, A., Kapitaniak, T., and Brindley, J.: Dynamics of coupled Lorenz systems and its geophysical implications, Physica D, 98 , 594-598, 1996.

Tziperman, E., Cane, M. A., and Zebiak, S. E.: Irregularity and Locking to the Seasonal Cycle in an ENSO Prediction Model as Explained by the Quasi-Periodicity Route to Chaos, J. Atmos. Sci., 52(3), 293-306, 1995.

Wembe, E. T. and Yamapi, R.: Chaos sychronization of resistively coupled Duffing systems: Numerical and experimental investigations, Commun. Nonlinear Sci., 14, 1439-1453, 2009.

Yu, Y. H., Kwak, K., and Lim, T. K.: On-Off intermittency in an experimental synchronization process, Phys. Lett. A, 198, 34 $38,1995$. 